\title{
Correlação entre a distribuição de epicentros e a magnitude de terremotos ocorridos nos Andes de 2003 e 2018
}

\section{Correlation between epicenter distribution and magnitude of Andean earthquakes from 2003 to 2018}

João Henrique Rettore Totaro

Geólogo, professor do Departamento de Geografia da PUC Minas Professor do Programa de Pós-graduação em Geografia - Tratamento da Informação Espacial da PUC Minas, Brasil joaorettore@gmail.com

\section{Resumo}

Segunda etapa de projeto de investigação da lei de Gutenberg-Richter (1949) para a relação entre magnitude e frequência de terremotos, este trabalho traduz os resultados de uma investigação feita sobre os sismos andinos, como registrados na base de dados do USGS, entre 2003 e 2018 . O recorte espacial contempla toda a faixa tectônica ativa na margem $\mathrm{W}$ da América do Sul, mas procura distinguir, entre os fatores que delimitam a análise, não apenas os contatos tectônicos mas também a geometria da margem continental. Além de apontar grande variação na frequência de sismos ao longo da faixa estudada, também se oferece um modelo alternativo para a segmentação dos clusters de epicentros associados a sismos mais profundos.

Palavras-chave: terremotos andinos; frequência de ocorrência de sismos; magnitude

\begin{abstract}
As the second stage of an investigation of the Gutenberg-Richter (1949) law for the relationship between magnitude and frequency of earthquakes, this work translates the results of a research carried out on Andean earthquakes, as recorded in the USGS database, occurred between 2003 and 2018. The geographical frontiers of the sample cover the whole of the active tectonic region on the South American $\mathrm{W}$ bank, but seeks to distinguish, among the factors that delimit the analysis, not only the tectonic contacts as well as the geometry of the continental margin. In addition to pointing out a great variation in the frequency of earthquakes over the studied range, an alternative model is also offered for the segmentation of epicenter clusters associated with deeper earthquakes.
\end{abstract}

Keywords: Andean earthquakes; earthquake frequency; magnitude

\section{INTRODUÇÃO}

Alguns dos principais descritores de terremotos são associados a seus parâmetros focais, como a localização do hipocentro (latitude, longitude e profundidade), componentes do falhamento e da cinemática do movimento, além da magnitude e do instante em que o sismo ocorreu (STEIN; WYSESSION, 2003). A identificação desses elementos, tornada possível especialmente a partir da integração de estações sismológicas em rede mundial, oferece informações sobre a estrutura interna do 
planeta e sobre os processos nele ativos (UDÍAS; BUFORN, 1991), além de permitir a construção de modelos heurísticos do comportamento sísmico de regiões específicas cuja confiabilidade é cada vez maior, o que tem reflexos incontestáveis na elevação dos níveis de segurança das atividades humanas nessas áreas.

A heterogeneidade natural que caracteriza a distribuição espaçotemporal de eventos sísmicos, mesmo em faixas de idêntico caráter tectônico, pode ser reduzida a tendências de comportamento que indicam a grande complexidade dos sistemas geológicos de larga escala. Uma vez que os sismos não ocorrem a intervalos regulares, é preciso buscar extrair de grandes massas de dados correlações entre as variáveis que controlam a mecânica dos terremotos; tanto mais extensos os registros detalhados, mais sustentação se oferece às hipóteses de trabalho e a sua verificação. Dessa maneira, variações na frequência e na magnitude de sismos revelam padrões temporais determinados pela geodinâmica interna (ZALIAPIN; BEN-ZION, 2020): os mecanismos reológicos que controlam as condições de cessão rúptil dos materiais geológicos a condições de elevadas pressões e temperaturas operam de modo a produzir respostas descontínuas no tempo.

Em áreas tectônicas de caráter bem definido, como as dorsais mesooceânicas e zonas de subducção, há características particulares na relação entre magnitude, frequência e profundidade do hipocentro de sismos; os dois primeiros descritores estão relacionados pela equação de GutenbergRichter (1949), a qual é aplicável a diversos ambientes tectônicos e complementar às leis de OmoriUtsu (UTSU et al., 1995) e de Båth (B ̊̊TH, 1965) ${ }^{1}$. A considerar a variação na espessura da crosta sob os Andes entre 32km e 78km (BECK et al., 1996; RYAN et al., 2016) e a estrutura de uma zona de subducção (ZS), a faixa de profundidades a que a ruptura sismogênica pode ocorrer atinge algumas centenas de quilômetros, coincidindo com os limites físico-químicos do comportamento frágil dos materiais geológicos (STERN, 2004).

\section{SISMICIDADE MUNDIAL}

Os epicentros de terremotos são majoritariamente localizados nas proximidades de faixas de contato entre placas tectônicas (GUTENBERG; RICHTER, 1949; BÅTH; DUDA, 1979; SITHARAM et al., 2018), como ilustra o mapa 1 para $M>5.0$. Em virtude de se verificarem nessas regiões as condições necessárias à produção de terremotos, como a mudança brusca nas relações entre as tensões

1 Trata-se das três primeiras leis básicas da sismologia, estabelecidas empiricamente; uma quarta, estabelecida por Felzer-Brodsky (2006), trata da frequência de incidência de réplicas e ainda é objeto de intenso debate. 
estáticas normal $\sigma_{1}$ e cisalhante $\sigma_{3}$ no domínio rúptil (critério de ruptura de Coulomb) e a variação nas pressões litostática e de fluidos (KING; STEIN; LIN, 1994).

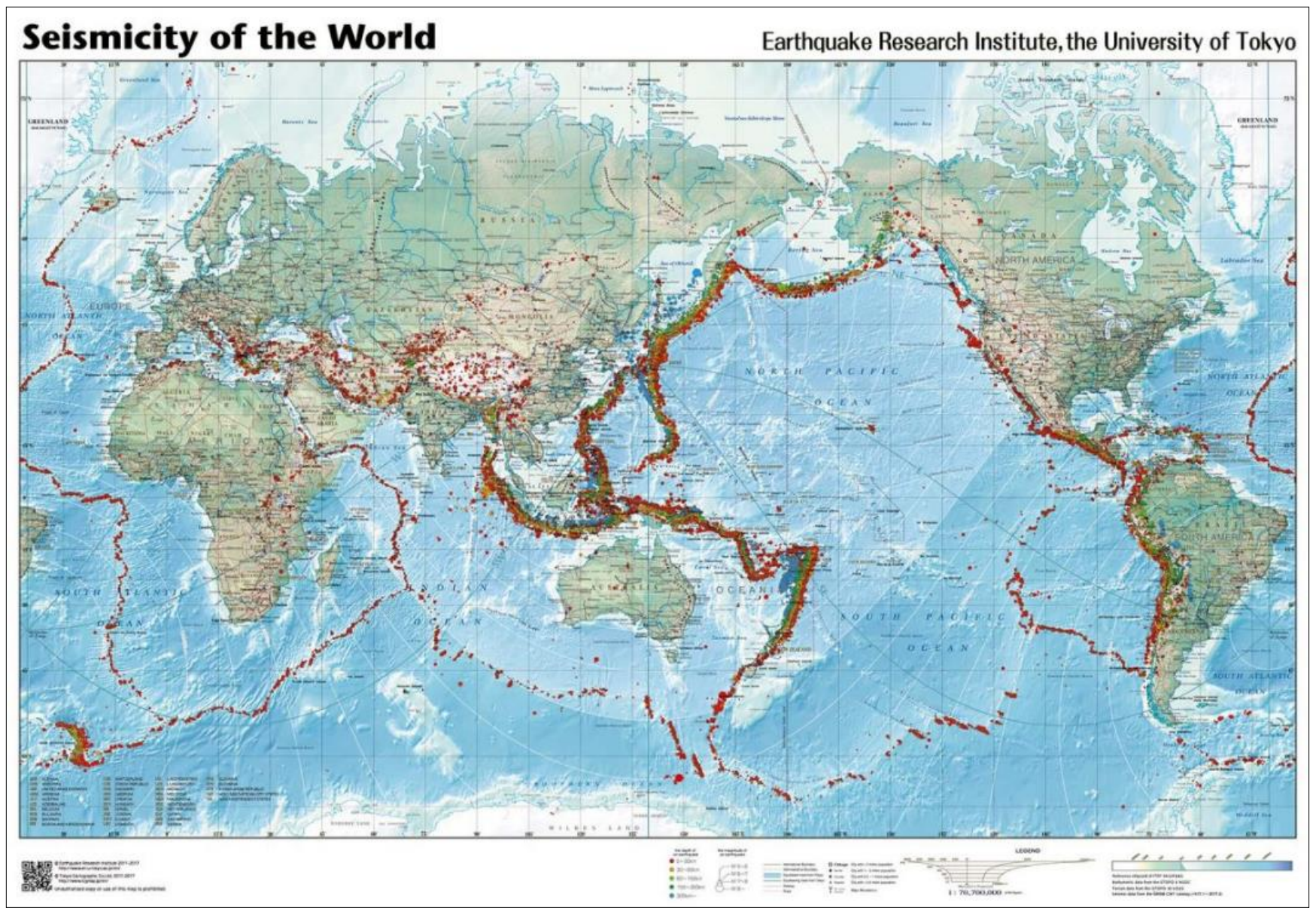

Mapa 1. Localização de epicentros de sismos de magnitude igual ou superior a 5.0 ocorridos entre 2011 e 2017 (ERI / Univ. Tokyo). Sismos de magnitudes mais baixas são dispersos em torno dessas faixas principais, que coincidem com os limites das placas tectônicas.

\section{CARACTERIZAÇÃO GERAL E HISTÓRICO SÍSMICO NOS ANDES}

A Cordilheira dos Andes expressa na geomorfologia a resposta ao encurtamento tectônico recente e contínuo da margem continental ocidental da placa da América do Sul, a qual se sobrepõe à placa oceânica de Nazca, que é consumida, por sua vez e nesse contato, em subducção (Figura 1). Trata-se de cinturão de dobramentos e falhamentos tornado ainda mais complexo devido à conjugação de processos tectônicos, magmáticos e acrecionais (TURIENZO et al., 2012). Há modelos que preveem diferentes graus para esse encurtamento (SOBOLEV; BABEYKO, 2005; GERYA, 2010) tomando como variáveis a espessura da crosta, o módulo e a direção do vetor de convergência do conjugado das duas placas e o coeficiente de atrito entre elas. 


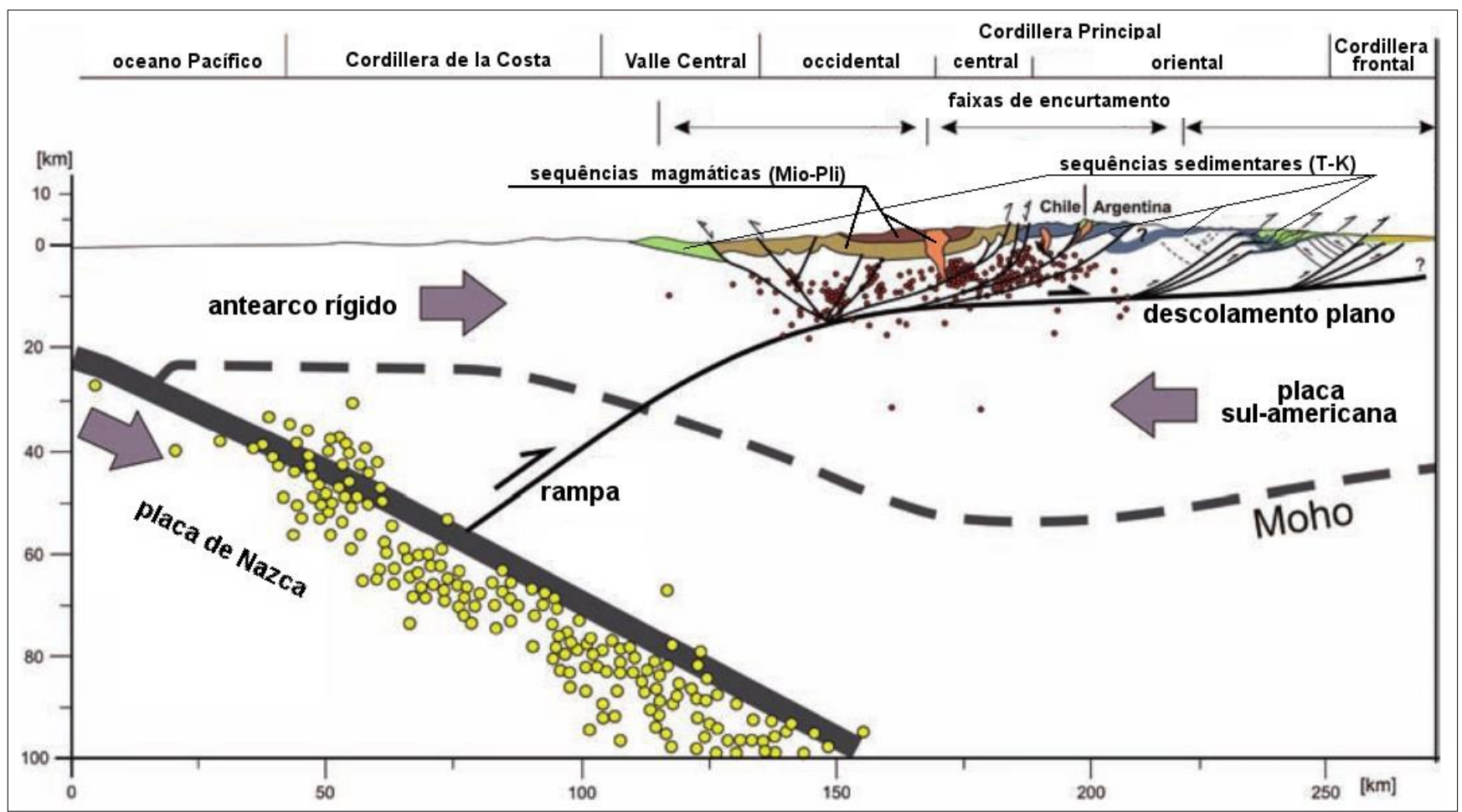

Figura 1. Seção proposta para o sistema tectônico Nazca - América do Sul próximo do paralelo 34S (Turienzo et al. 2010:327, adaptado) e segundo modelo mais conservador de encurtamento da margem continental. Os círculos representam, próximo da placa de Nazca e das faixas de cavalgamento, epicentros de terremotos ligados a mecanismos sismogênicos distintos.

A convergência entre a placa da América do Sul, a E, e as placas de Nazca e da Antártida, a W e a SW, respectivamente, cria extensa ZS limitante da faixa continental da América do Sul, onde se concentra grande parte dos epicentros de terremotos no Pacífico Oriental (ROTHÉ, 1969; DÉVERCHÈRE, 1988; METOIS, 2009; ZALIAPIN; BEN-ZION, 2020). Uma busca no catálogo eletrônico do USGS pelos registros de terremotos ocorridos numa janela em torno da América do Sul lista 5862 eventos de $\mathrm{M} \geq 5.0$ entre 1900 e 1999, como mostra o mapa 2; destaca-se a franca sobreposição de pontos de várias magnitudes numa faixa relativamente estreita e coincidente com as bordas de placas tectônicas.

Concentramo-nos, aqui, apenas na faixa que compreende os Andes, que foi dividida em três setores segundo a) o contato entre placas e b) o ângulo entre o vetor de convergência interplacas e a linha de costa da América do Sul. O primeiro segmento segue desde a fronteira entre Panamá e Colombia até a fronteira entre Peru e Chile; o segundo, desse último ponto até o paralelo 46S; o terceiro, daí até o paralelo 58S. Dessa forma, temos três setores - setentrional, central e meridional - aos quais se pode associar uma hipótese de diferença de comportamento sísmico graças à combinação desses fatores tectônico e geométrico. 


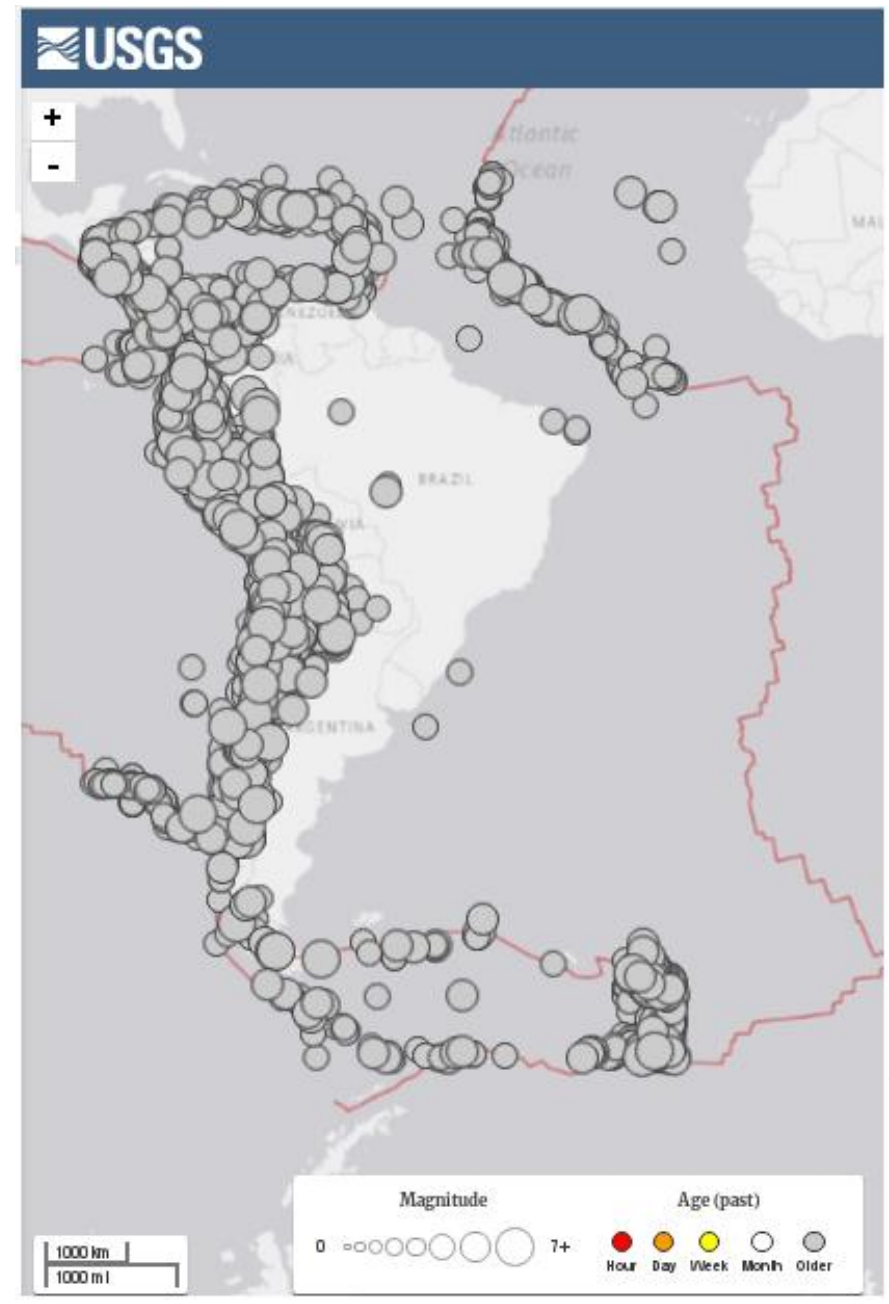

Mapa 2 - Localização de epicentros de terremotos ocorridos na América do Sul, como constantes da base de dados do USGS, de jan/1900 a dez/1999. Os sismos representados pelos círculos perfazem um total de 5862 eventos, todos de $M \geq 5$.0. Nota-se claramente que a faixa correspondente à ZS não restringe os epicentros a suas adjacências, mas que estes se projetam ainda mais para $\mathrm{E}$ sobre a placa continental por serem originados a profundidades cada vez maiores.

\section{BASES DE DADOS EM SISMOLOGIA}

Há diversas fontes de informação sobre terremotos em sites institucionais ou governamentais, as quais comportam características distintas em termos de concepção e objetivos, metodologias de trabalho e tipologia de dados, alcance cronológico das informações oferecidas e projetos associados. Essa diversidade exige, frequentemente, que se consultem mais de uma dessas fontes em caráter de complementaridade (OGATA; KATSURA, 1993). Entre alguns dos mais amplos em relação ao intervalo temporal de registro e de diversidade de informações técnicas, destacam-se:

IRIS (Incorporated Research Institutions for Seismology) - além de fácil acesso a larga base de dados sobre eventos sísmicos ocorridos desde a década de 1970 até os mais recentes (o que inclui a primeira série de informações colhidas pela missão InSight em Marte), comporta mapas, textos técnicos e grande diversidade de informações científicas em sismologia. 
www.iris.edu/hql

USGS (United States Geological Survey) - oferece mapas, acesso a extensa base de dados e informações técnicas e científicas sobre terremotos.

earthquake.usgs.gov

NOAA (National Centers for Environmental Information) - compreende catálogos de séries históricas e atuais sobre terremotos significativos (especialmente anteriores a 1960), além de informações sobre riscos sísmicos.

www.ngdc.noaa.gov/hazard/earthqk.shtml

EMSC (European Mediterranean Seismological Center) - oferece mapas e informações técnicas e científicas sobre terremotos globais e, em particular, sobre o Mediterrâneo.; responde pela coordenação de diversos projetos de cooperação em sismologia.

www.emsc-csem.org/

Escolheu-se a base de dados do USGS pela facilidade de geração de listas de informações, em formato .CSV (de imediata importação para planilhas eletrônicas), sobre sismos ocorridos em qualquer parte do mundo. O intervalo de tempo foi fixado entre 2003 e 2018, compreendendo 15 anos de amostragem nesta etapa preliminar de teste de hipóteses; ademais, a magnitude dos terremotos, neste estudo, é de $\mathrm{M} \geq 1$, excluindo, portanto, microssismos. Apenas eventos naturais foram selecionados, o que exclui tremores antropogênicos de qualquer natureza.

\section{DISTRIBUIÇÃO ESPACIAL DE EPICENTROS}

Além da segmentação em três domínios tectônico-geométricos, as informações sismológicas foram classificadas segundo faixas de profundidade, o que, por um lado, obedece à identificação de níveis estruturais (MATTAUER, 1980; BOUCHEZ; NICOLAS, 2018), que correlacionam a reologia dos materiais à recíproca entre pressão confinante e gradiente térmico. Toma-se, para toda a linha ocidental da América do Sul, $\sigma_{1}$ de direção predominante E-W, com deflexões locais decorrentes das heterogeneidades composicionais inerentes aos litotipos integrantes da conformação regional e das descontinuidades estruturais já instaladas nessas sequências litológicas. 


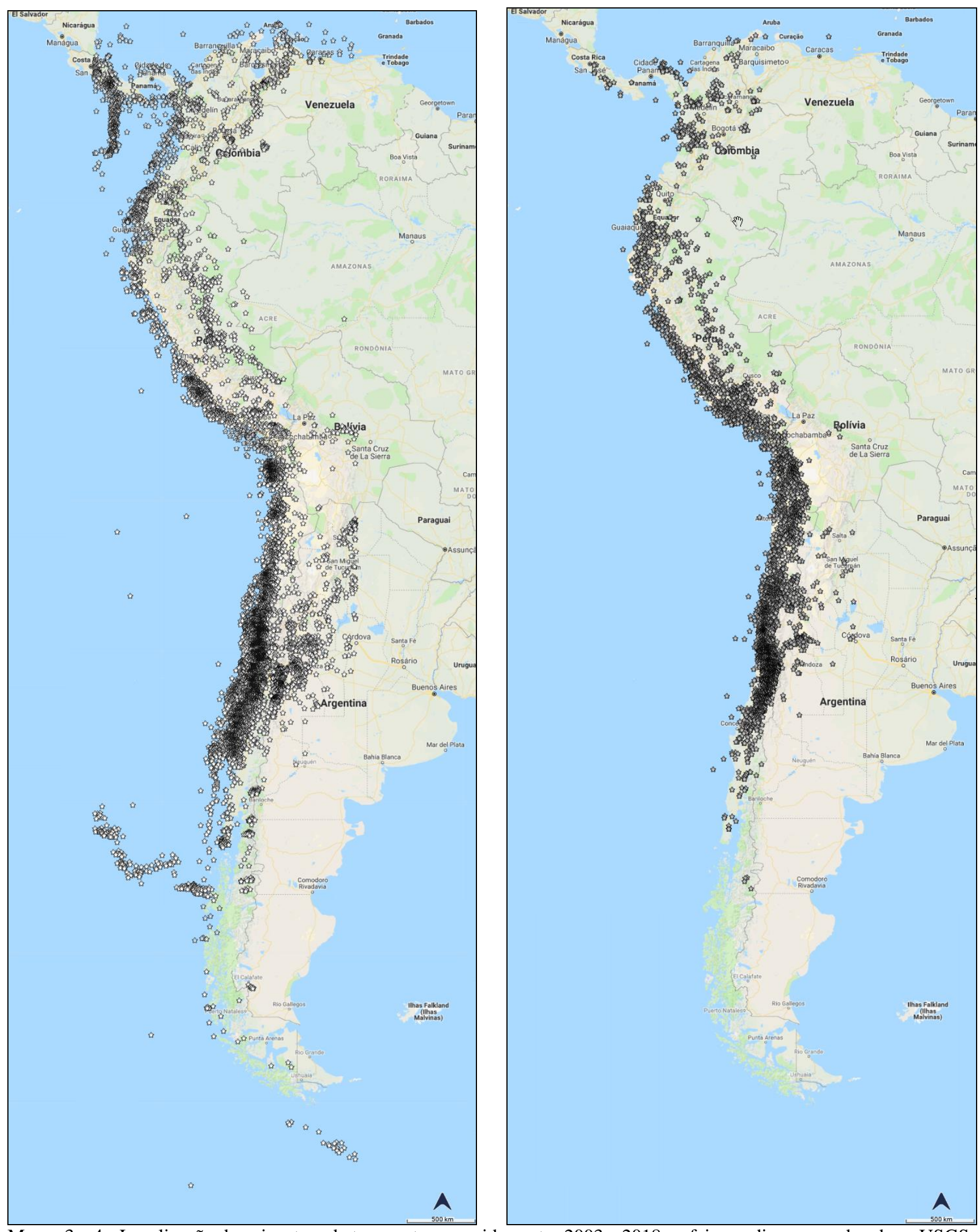

Mapas 3 e 4 - Localização de epicentros de terremotos ocorridos entre 2003 e 2018 na faixa andina, segundo a base USGS, em intervalos de profundidade de $0.00 \mathrm{~km}$ a $49.99 \mathrm{~km}$ e de $50.00 \mathrm{~km}$ a $99.99 \mathrm{~km}$, respectivamente. Nota-se claro estreitamento do segundo cluster em relação à sensível dispersão do primeiro, o que indica restrição das condições sismogênicas a profundidades crescentes. 
A reduzida pressão confinante permite maior dispersão dos epicentros rasos $(0 \mathrm{~km}-49.99 \mathrm{~km})$, que ocupam não apenas a faixa imediatamente adjacente à $\mathrm{ZS}$, mas também de distribuem subparalelamente a ela, em particular nas áreas continentais, até para além da janela de amostragem limitada pela longitude 64W. Essa faixa continental que coincide com o arco ocidental da América do Sul indica, por certo, a propagação de stress que se resolve na forma de strain rúptil sob menor pressão litostática, mas que também assume, em maiores profundidades, feições de deformação plástica (MATTAUER 1980).

Outro ponto que merece destaque no tratamento dos dados é a variação na densidade de sismos entre os terços setentrional, intermediário e meridional da costa $\mathrm{W}$ da América do Sul: a porção intermediária é claramente caracterizada por maior incidência de terremotos rasos de várias magnitudes, o que eleva sobremaneira os riscos geológicos direto e indiretos, imediato e mediatos associados a esses eventos. Esse padrão tende a persistir nas demais classes de profundidade, como se verá adiante, identificando a porção intermediária como a de mais elevada sismicidade para todas as profundidades a que os sismos podem ser originados. Está em desenvolvimento o estudo da correlação fina entre a profundidade, a magnitude e o tempo nessa mesma base de dados, o que levará a considerações mais próprias sobre o comportamento de zonas tectônicas de grande escala.

É interessante observar, em relação à dispersão dos pontos correspondentes aos sismos mais rasos, o estreitamento da faixa em que se distribuem os epicentros de terremotos produzidos entre $50 \mathrm{~km}$ e 99.99km. Em outras palavras, há uma espacialização nítida dos epicentros nesta segunda classe, de sorte que, no período de amostragem, há sensível regularidade na localização de sismos nesse intervalo de profundidades. Por certo, é igualmente importante fazer uma correlação entre os descritores desses eventos a fim de se determinar a suscetibilidade à ocorrência de sismos de maior intensidade nessa faixa; tratamento mais refinado da mesma base de dados está em processo, envolvendo o cruzamento dos fatores aqui usados para determinar, para uma mesma região, a suscetibilidade à ocorrência de sismos mais intensos. 


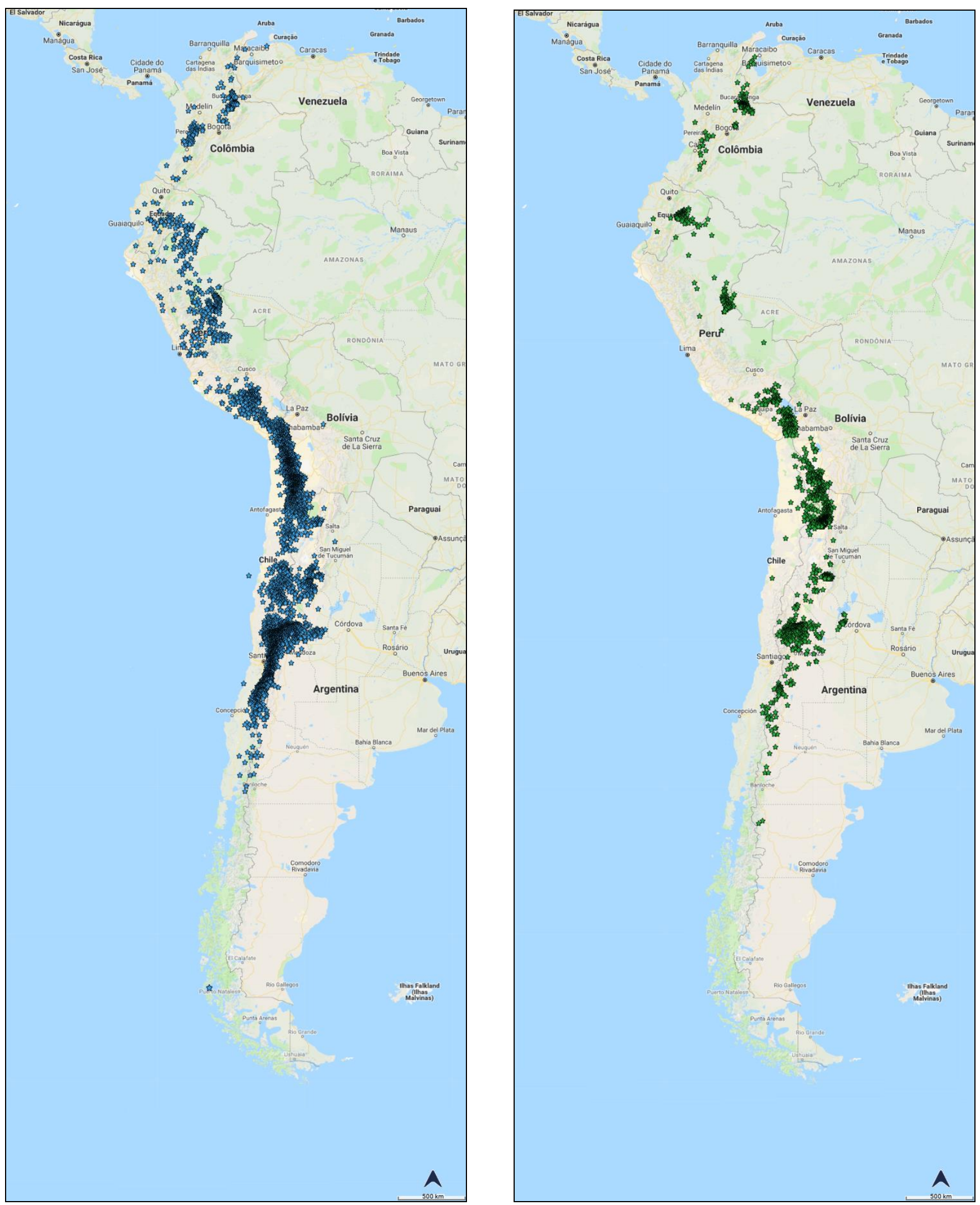

Mapas 5 e 6 - Localização de epicentros de terremotos ocorridos entre 2003 e 2018 na faixa andina, segundo a base USGS, em intervalos de profundidade de $100.00 \mathrm{~km}$ a $149.99 \mathrm{~km}$ e de $150.00 \mathrm{~km}$ a $199.99 \mathrm{~km}$, respectivamente. Ambas classes tendem a se concentrar na faixa longitudinal intermediária dos Andes, indicando que as condições sismogênicas se sustentam mais expressivamente nessa região às profundidades focais. 
Nos mapas 5 e 6, diversos aspectos da distribuição espacial dos epicentros chamam imediatamente a atenção. Em primeiro lugar, sobressai ainda mais claramente a predominância da sismicidade do terço intermediário da faixa $\mathrm{W}$ da América do Sul, mas esse mesmo segmento é aqui ainda subdividido em outras três partes de sismicidade também distinta. Nesse nível, a porção intermediária é de muito mais baixa sismicidade que as demais, o que levou Metois (2012) a propor um modelo de acoplamento e cessão segundo o qual faixas de maior atrito estático seriam alternadas com outras de menor aspereza: a liberação da energia mecânica armazenada nas faixas mais ásperas liberaria ondas de choque percebidas como sismos. Entretanto, esse modelo implica a) que as isotermas são horizontais e não acompanham a placa em subducção e b) que uma placa litosférica em subducção seja conduzida como uma unidade às profundidades em que a fusão é completa.

Uma alternativa que não apenas é reologicamente correta mas também responde pela distribuição de epicentros em linhas E-W nos mapas 5 a 8 sugere que a placa de Nazca seja seccionada de maneira a se verificarem espaços distintos de acúmulo de energia mecânica. Essa hipótese é corroborada pelo fato de que a porção medial do segmento intermediário da base de dados é limitada a $\mathrm{N}$ e a S pela disposição de epicentros alinhados em direção subperpendicular ao eixo principal do mesmo segmento e coincidentes com possíveis faixas de rasgamento da placa; a Figura 2 abaixo ilustra a situação.

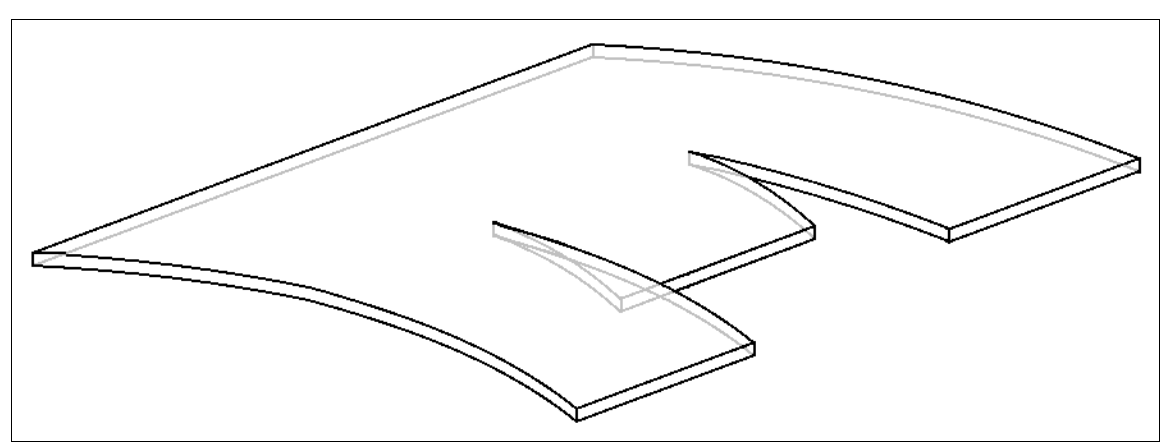

Figura 2. Esboço da configuração hipotética da placa de Nazca sob a América do Sul, a partir da distribuição de epicentros de terremotos cujos focos são mais profundos que 100km. Os três segmentos têm inclinações diferentes, o que leva a frequências distintas de ocorrência de sismos a profundidades maiores.

Os mapas 5 e 6 mostram nítida concentração dos epicentros mais distante da linha de costa em decorrência do aumento progressivo da profundidade da placa em subducção para E. Ainda que ocorram sismos de várias profundidades nas mesmas faixas, os mais rasos tendem, por certo, a ser menos atenuados em termos de dissipação da energia mecânica por eles liberada. 

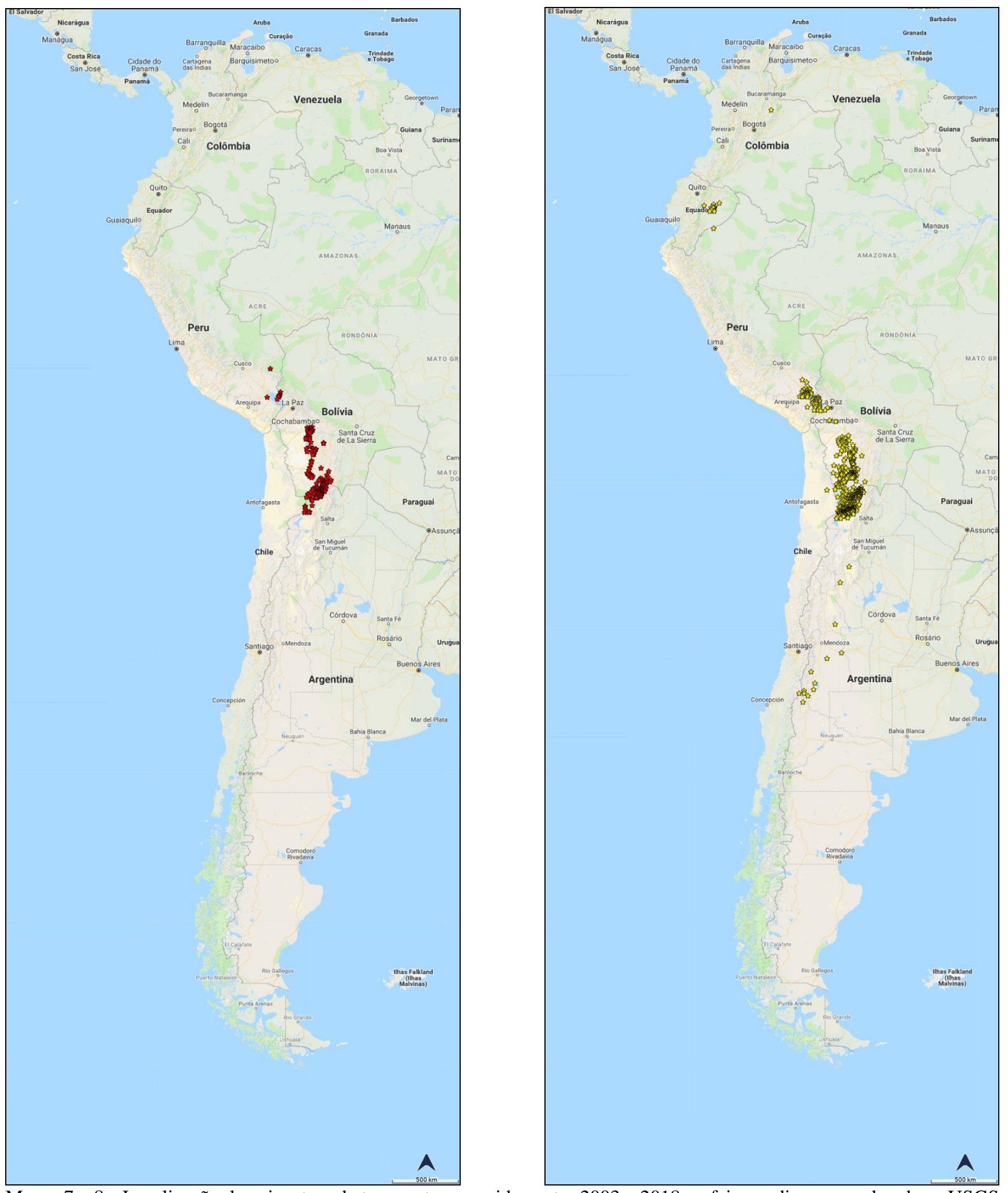

Mapas 7 e 8 - Localização de epicentros de terremotos ocorridos entre 2003 e 2018 na faixa andina, segundo a base USGS, em intervalos de profundidade de $200.00 \mathrm{~km}$ a $249.99 \mathrm{~km}$ e de $250.00 \mathrm{~km}$ a $299.99 \mathrm{~km}$, respectivamente. Muito mais limitados, os clusters se concentram na porção superior do terço intermediário, de sorte a corroborar o modelo proposto na Figura 2: as demais frações da placa de Nazca seriam, pela mesma proposta, subductadas em ângulo mais alto do que o obtido acima por meio de relação trigonométrica. 
Nos mapas 7 e 8, o intervalo de profundidades compreendido entre 200 e 300km está associado a ainda mais intensa restrição distribucional dos epicentros, que se localizam no setor superior do terço intermediário da região em apreço. Por certo, trata-se também, nesses dois subconjuntos de dados, de sismos muito menos comuns: a frequência absoluta dos eventos, como se observa do primeiro ao último mapas, é inversamente proporcional à profundidade do hipocentro. Também se afastam ainda mais para $\mathrm{E}$ as duas classes de dados, ainda em verificação ao disposto na Figura 2 acima: apenas uma das frações da placa de Nazca estaria apta a produzir sismos por atrito ou ruptura à distância que se encontra da ZS.

Demonstra-se, pelo método empregado, uma correlação inversa entre profundidade e frequência de ocorrência de sismos na região andina, o que atesta, uma vez mais, a estreita dependência entre nível estutural e tipologia de deformação: o caráter rúptil, garantido pelo critério de Coulomb, é preferencialmente associado a profundidades mais rasas, enquanto a deformação assume feições essencialmente plásticas a grandes profundidades. Apenas a elevada taxa de deformação e o considerável módulo de stress compressivo que caracterizam a região possibilitam a produção de sismos em grandes profundidades, como apontam os mapas 7 e 8.

Pelo mesmo procedimento, é possível inferir a inclinação aproximada da placa de Nazca sob a sul-americana, tomando-se o segmento em projeção horizontal como correspondente à distância entre a ZS e a posição dos epicentros mais profundos do mapa $8(300 \mathrm{~km})$. Um cálculo simples oferece um valor de cerca de $30^{\circ} \mathrm{E}$ de inclinação para a fração da placa de Nazca que está sob a sul-americana. Até a profundidade máxima da amostragem apresentada no mapa 8, veja-se, portanto, que ainda há cessão rúptil do material litosférico e consequente deflagração de terremotos; a atenuação das ondas de choque, por certo, tende a tornar ainda menos intensos esses eventos, além de constituir um perfil sismográfico característico ao relacionar os valores de energia transmitida por ondas de corpo sólido em relação às transversais (LEI, 2018).

A tabela apresentada a seguir oferece, em termos objetivos, uma perspectiva geral sobre a distribuição de eventos sísmicos nos Andes em relação ao tempo, à profundidade e à magnitude. Chamam a atenção:

a. a preponderância absoluta da faixa central quanto à frequência de ocorrência de terremotos, enquanto a porção meridional os têm pouquíssimos;

b. o fato de que a maioria dos sismos ocorre, em todas as faixas, a baixa profundidade (0km$49.99 \mathrm{~km}) \mathrm{e}$

c. a predominância de sismos de $\mathrm{M}=3.0$. 


\begin{tabular}{|c|c|c|c|c|c|c|c|c|c|c|}
\hline \multicolumn{11}{|l|}{ setentrionais } \\
\hline & período & 1 & 2 & 3 & 4 & 5 & 6 & 7 & 8 & total \\
\hline & & 897 & 1185 & 847 & 506 & 651 & 909 & 1094 & 370 & 6459 \\
\hline & profundidade & 1 & 2 & 3 & 4 & 5 & 6 & 7 & 8 & \\
\hline & & 3205 & 1147 & 820 & 1128 & 90 & 11 & 26 & 32 & \\
\hline & magnitude & 1 & 2 & 3 & 4 & 5 & 6 & 7 & & \\
\hline & & 370 & 525 & 4828 & 644 & 81 & 11 & 0 & & \\
\hline
\end{tabular}

\begin{tabular}{|l|l|l|l|l|l|l|l|l|l|l|}
\hline centrais & \multicolumn{1}{|l|}{} \\
\cline { 2 - 11 } & período & 1 & 2 & 3 & 4 & 5 & 6 & 7 & 8 & total \\
\hline
\end{tabular}

\begin{tabular}{|l|l|l|l|l|l|l|l|l|}
\hline profundidade & 1 & 2 & 3 & 4 & 5 & 6 & 7 & 8 \\
\hline & 12554 & 3431 & 4539 & 1706 & 770 & 150 & 13 & 0 \\
\hline
\end{tabular}

\begin{tabular}{|l|l|l|l|l|l|l|l|}
\hline magnitude & 1 & 2 & 3 & 4 & 5 & 6 & 7 \\
\hline
\end{tabular}

meridionais

\begin{tabular}{|l|r|r|r|r|r|r|r|r|r|}
\hline período & 1 & 2 & 3 & 4 & 5 & 6 & 7 & 8 & total \\
\cline { 2 - 11 } & 14 & 5 & 9 & 7 & 2 & 8 & 15 & 1 & 61 \\
\hline
\end{tabular}

\begin{tabular}{|r|r|r|r|r|r|r|r|r|r|}
\hline profundidade & 1 & 2 & 3 & 4 & 5 & 6 & 7 & 8 \\
\hline
\end{tabular}

\begin{tabular}{|l|l|l|l|l|l|l|l|}
\hline magnitude & 1 & \multicolumn{1}{l|}{2} & \multicolumn{1}{l|}{3} & \multicolumn{1}{l|}{4} & 5 & 6 & 7 \\
\hline
\end{tabular}

total

29680

Tabela 1. Terremotos ocorridos nos Andes entre 2003 e 2018, segundo a base de dados do USGS. A segmentação dos dados pretende demonstrar a hipótese de que há faixas de comportamento sísmico distinto em função de configurações igualmente diversas.

Todos esses elementos são interessantes indicadores de mecanismos sismogênicos, mesmo que não contemplem microssismos: em primeiro lugar, percebe-se que a relação entre magnitude e frequência não é decrescente, mas assemelha-se a uma sinusoide assimétrica de elevada curtose; isso indica que os terremotos andinos são deflagrados por processos cuja associação é mais favorável a baixas profundidades e associados a magnitudes da ordem de $M=3.0$. Por certo, a intensidade desses eventos deve ainda ser estudada a partir da verificação da frequência com que a magnitude do sismo acompanha a profundidade de seu hipocentro. 
Em forma gráfica e após tratamento simplificado, os dados dispostos acima indicam, em blocos:

a. em termos de frequências relativas ${ }^{2}$ : os três gráficos mostram, em linhas gerais, alguma identidade entre as três faixas representadas em cada um

a.1. por período: os perfis dos três setores são semelhantes, guardando correlação mediana alta (0.5745 a 0.6762) entre si. É peculiar o fato de que o mínimo local intermediário na frequência de sismos na faixa setentrional tenha ocorrido no período de 09/2009 a 08/2011, anterior ao biênio observado nas duas outras faixas;

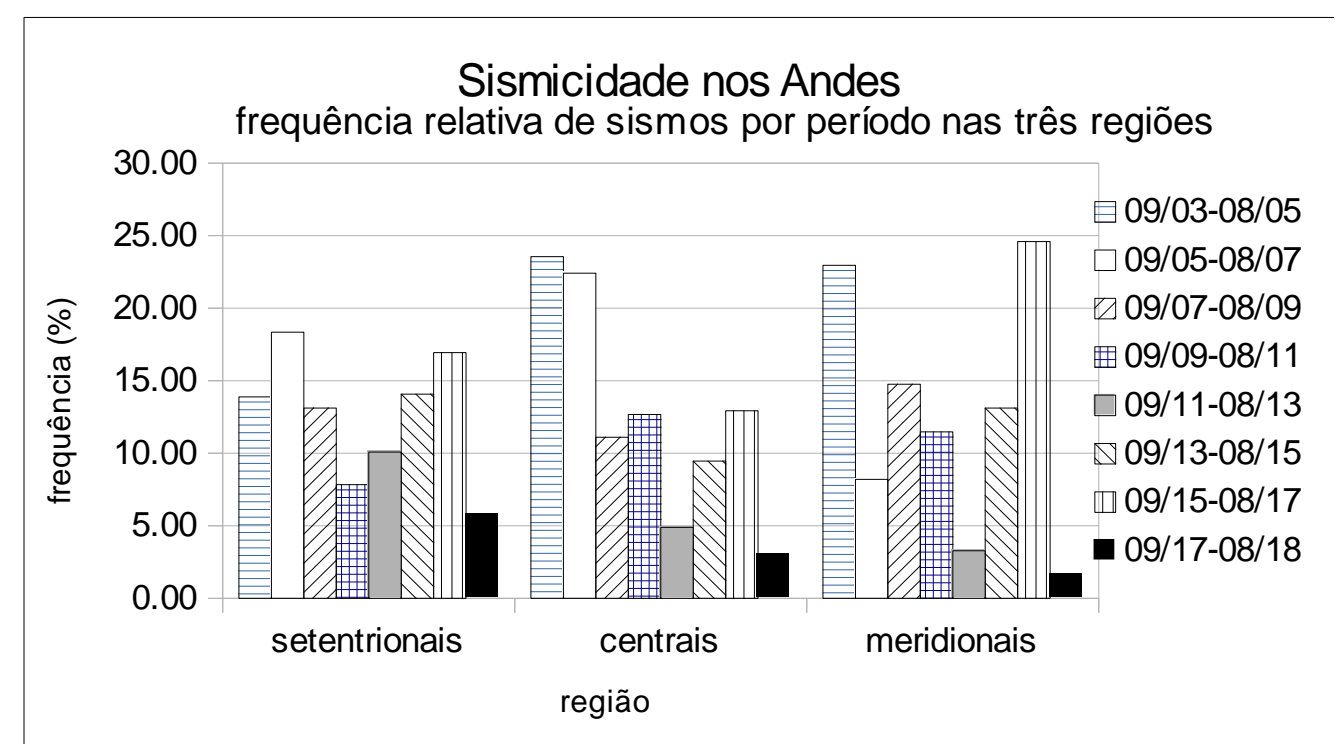

Gráfico 1. Frequências relativas calculadas para os três segmentos principais em relação ao período de 2003 a 2018 , destacando-se, para os três subconjuntos, a ocorrência de um mínimo local entre 2009, para o primeiro, e 2013, para os dois últimos.

a.2. por faixa de profundidade: há excelente correlação entre os setores setentrional e central (0.9611), mas ela é menos favorável entre ambos e o setor meridional, em que sobressai quase exclusivamente a faixa mais rasa; esse dado corrobora a hipótese de que o comportamento sísmico dos Andes é setorizado também para o fator profundidade de foco (TURIENZO et al., 2010);

2 Observe-se que os dados correspondentes à região dos Andes Maridionais, como aqui delimitada, são muito menos numerosos (i.e., sua significância é menor) que os referentes às frações setentrional e central. 


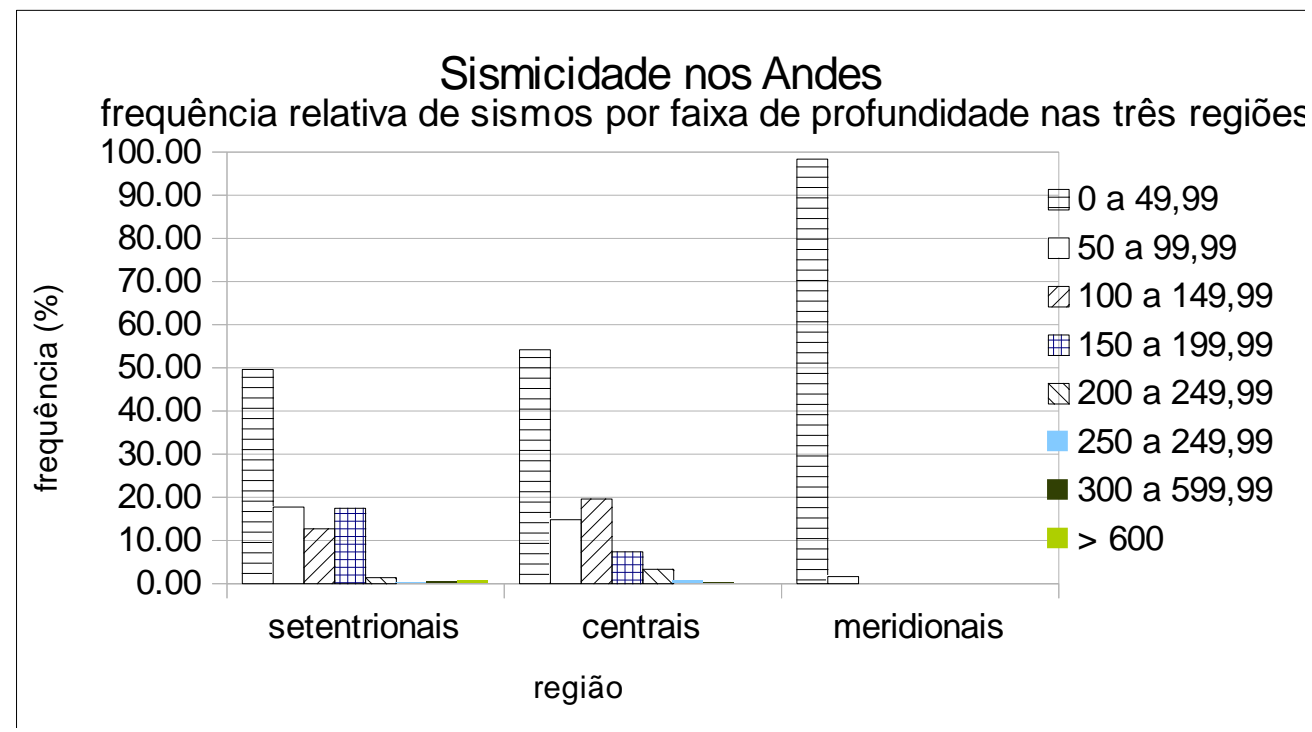

Gráfico 2. Frequências relativas calculadas para os três segmentos principais em relação às faixas de profundidade de hipocentros e entre 2003 a 2018, destacando-se, para os três subconjuntos, uma tendência decrescente nas proporções de sismos a profundidades crescentes, apesar de essa relação não ser linear em função, naturalmente, das idiossincrasias de cada segmento, como discutido acima.

a.3. por intervalo de magnitude: mesmo ao exame do gráfico é possível notar que a melhor correlação é feita entre as faixas setentrional e meridional em função da predominância, em ambas, de eventos cuja potência está situada entre $M=4.0$ e $M=4.9$, com reduzida participação das demais classes de magnitude. Essa avaliação deve ainda ser tomada, por certo, conjugada com os fatores tempo e profundidade para se determinar a suscetibilidade à ocorrência de terremotos de maiores intensidades em qualquer dessas regiões.

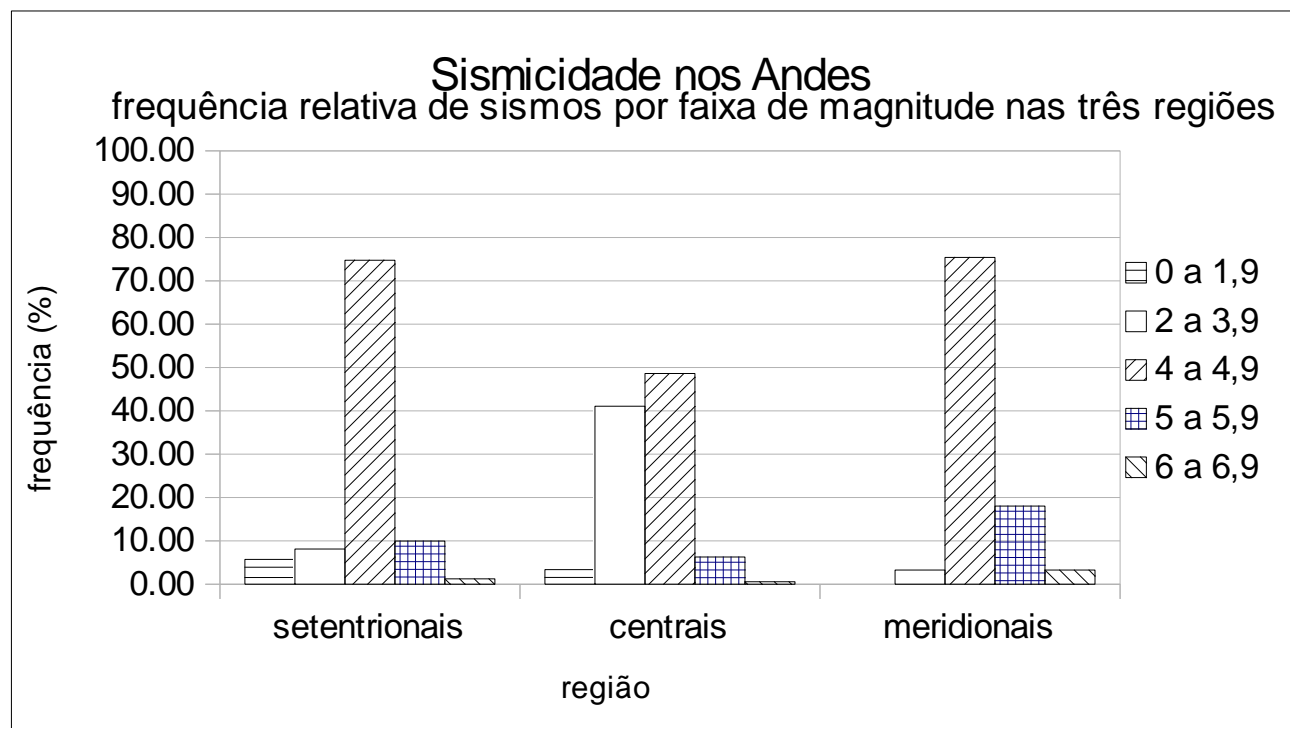

Gráfico 3. Frequências relativas calculadas para os três segmentos principais em relação às faixas de magnitude dos eventos sísmicos e no período de 2003 a 2018; uma vez mais, os três subconjuntos da mesma ZS funcionam de maneira similar, ainda que o terço meridional seja muito menos ativo que os outros dois. 


\section{b. em termos de frequências absolutas:}

b1. por período: sobressai a faixa central, dominante sobre as demais em todos os intervalos temporais. Já assinalada, a elevada correlação entre as frequências de ocorrência de sismos nas três zonas (ainda que não perceptível, na escala do gráfico, para a faixa meridional, mas sim, na tabela 1 acima) afirma que os Andes se comportam como testemunho de processos de mesma natureza decorrentes de subducção. Todo o sistema mostra, por certo, alguma flutuação na frequência de eventos sísmicos que merece ser ainda estudada com séries temporais mais abrangentes, procurando-se identificar possíveis padrões temporais de distribuição de terremotos e buscar modelos que expliquem esse comportamento variável;

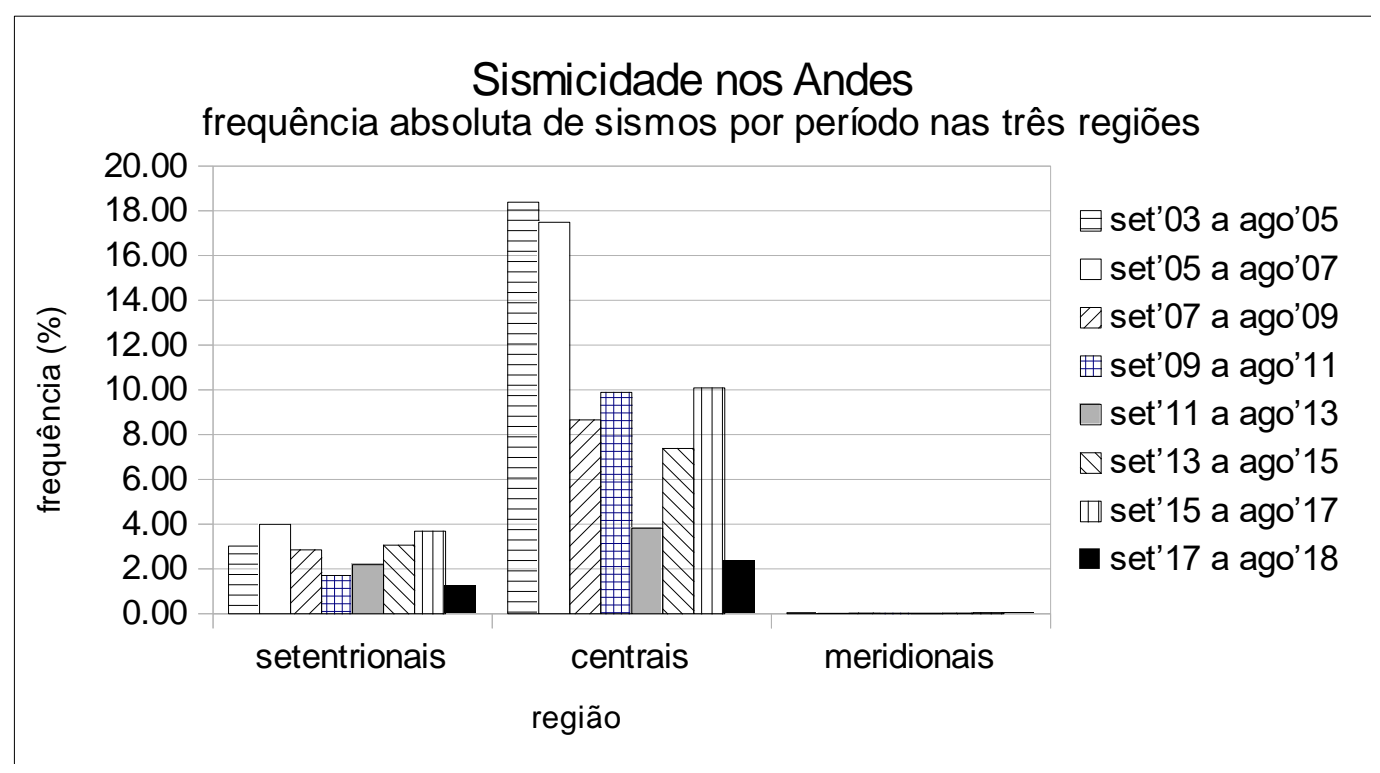

Gráfico 4. Frequências absolutas calculadas para os três segmentos principais em relação ao período de 2003 a 2018 . A partir do início do intervalo de amostragem, há tendência generalizada a redução na frequência de sismos nas duas primeiras faixas; a última é mais propriamente observada na tabela 1. Em toda a amostra, a sismicidade é absolutamente predominante na faixa central.

b2. por faixa de profundidade: mesmo que apresentem uma redução sistemática da frequência diante do aumento de profundidade (o que corrobora a prevalência das condições favoráveis ao comportamento rúptil em condições mais rasas), os máximos locais diferentes para os terços setentrional e central são muito interessantes. Sugerem, possivelmente, que a taxa mais elevada de subducção e o ângulo de entrada da placa de Nazca podem causar ruptura mais efetiva a profundidades menores (e, potencialmente, sismos mais intensos) na faixa central dos Andes; 


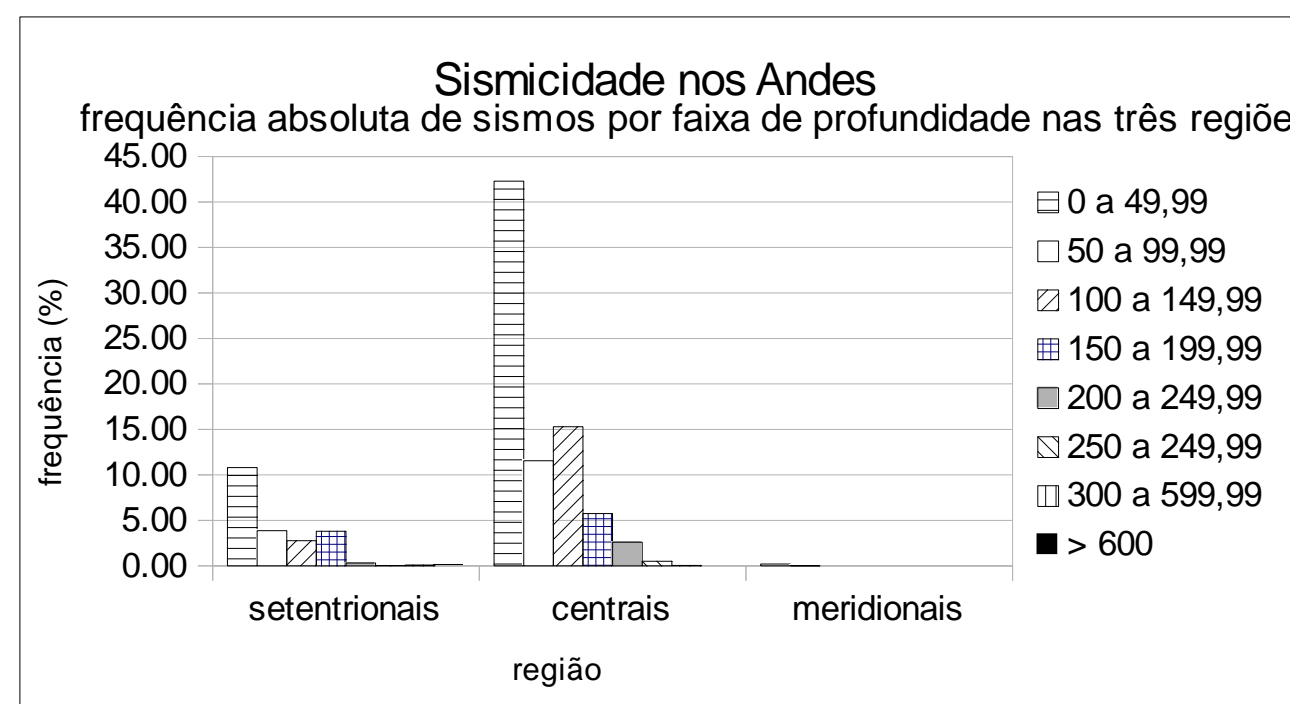

Gráfico 5. Frequências absolutas calculadas para os três segmentos principais em relação às faixas de profundidade, restritas ao período de 2003 a 2018. Mesmo que não se resolva numa curva continuamente decrescente, a relação entre essa frequência e a profundidade é inversa; uma vez mais, prevalece a faixa intermediária sobre as demais.

b3. por faixa de magnitude: a porção central, mais ativa em termos de incidência de sismos, é também aquela em que se pode observar mais claramente que, no intervalo de amostragem, a relação entre frequência e magnitude não é inversa. É importante notar que a fonte de dados é capaz de registrar eventos de magnitudes negativas (estes, sim, em altíssimo número), mas permanece ainda a questão da elevada proporção de eventos de $2.0 \leq \mathrm{M}<6.0$.

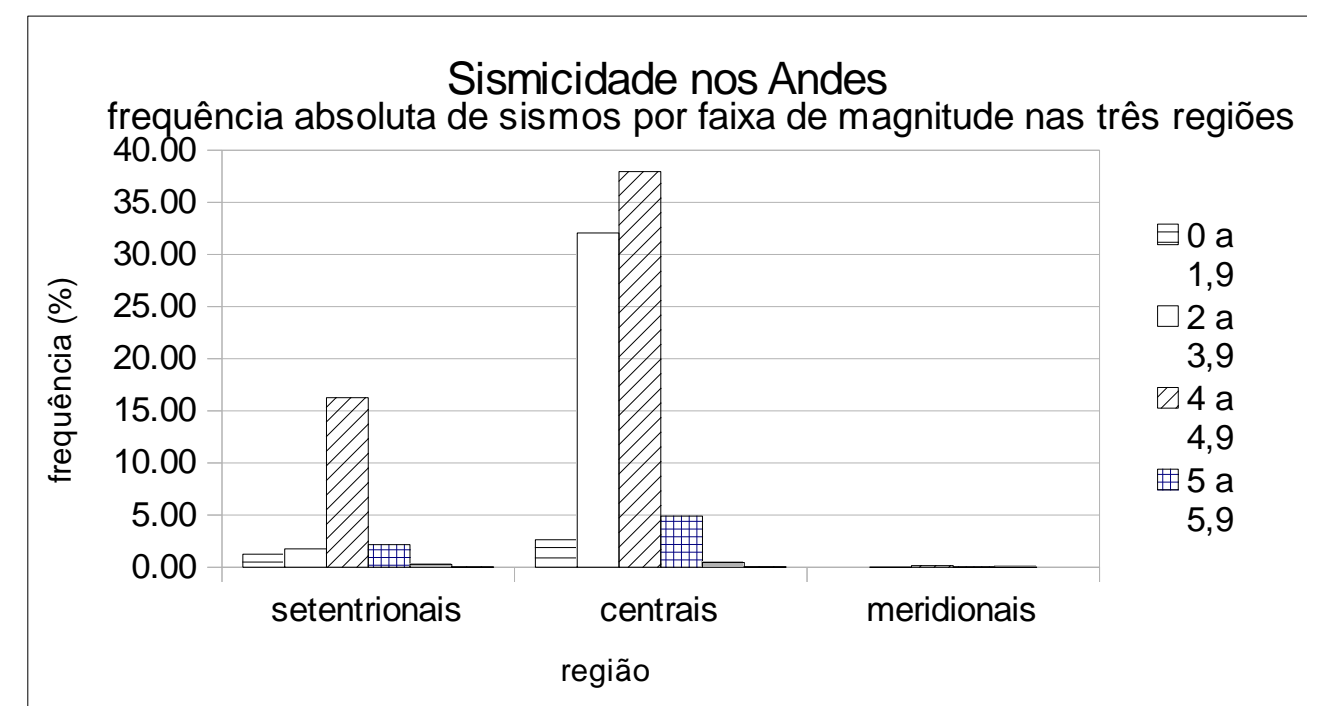

Gráfico 6. Frequências absolutas calculadas para os três segmentos principais em relação às faixas de magnitude no período de 2003 a 2018. A classe mais frequente nos Andes, 4.0 $\leq \mathrm{M}<5.0$, é saliente nas três seções, a última das quais é melhor observada na tabela 1. 


\section{CONCLUSÕES}

Em bases de dados muito amplas, como as mencionadas neste trabalho, o recorte espacial permite divisar particularidades de comportamento de sistemas tectônicos específicos, como se pôde demonstrar para a faixa andina. Alguns elementos teóricos ainda devem ser trazidos à avaliação mais abrangente dos resultados, mas serão melhor discutidos à luz de

a) expansão da base de dados dos Andes de modo a contemplar intervalo temporal mais amplo, o que pode corroborar o modelo sugerido neste trabalho para a fragmentação de uma placa em subducção,

b) comparação entre a faixa dos Andes e outras de mesma natureza tectônica, evidenciando similaridades e distinções entre elas que possam remeter aos fatores que respondem por sua configuração e evolução e

c) contraposição entre ZSs e áreas tectônicas de transcorrência e de formação de nova litosfera. Não apenas a distribuição espaçotemporal de focos de terremotos pode apontar aspectos estruturais e processuais inerentes a regiões específicas e compartilhados entre aquelas de mesma natureza, mas também corresponde a uma das melhores ferramentas a nossa disposição para inferir e modelar as unidades, as relações e os mecanismos de evolução litosférica.

A porção central dos Andes, como definida acima, é o espaço em que ocorre a maior parte dos sismos de várias magnitudes na faixa ocidental da América do Sul; também uma base de dados mais ampla permitirá, proximamente, estabelecer de maneira mais clara a relação entre profundidade do hipocentro e magnitude dos terremotos andinos. Nesse sentido e no escopo do projeto de investigação conduzido na PUC/Minas, as três linhas de trabalho já estão em desenvolvimento e devem oferecer reflexão complementar relevante. 


\section{REFERÊNCIAS}

BÅTH, MARKUS. Lateral inhomogeneities in the upper mantle. Tectonophysics 2: 483-514, 1965.

BÅTH, MARKUS; DUDA, SEWERIN J. Some aspects of global seismicity. Tectonophysics 54(1-2): T1-T8, 1979.

BECK, SUSAN L.; ZANDT, GEORGE; MYERS, STEPHEN C; WALLACE, TERRY C.; SILVER, PAUL G.; DRAKE, LAWRENCE. Crustal-thickness variations in the central Andes. Geology 24(5): 407-410, 1996.

DÉVERCHÈRE, JACQUES. Premiers résultats sur la sismicité de la Cordillère Blanche (Hautes Andes du Pérou central). Géodynamique 3(1-2): 45-55, 1988.

FELZER, K. R.; BRODSKY. E. E. Decay of aftershock density with distance indicates triggering by dynamic stresses. Nature 441:735-8, 2006.

GERYA, TARAS. Introduction to numerical geodynamic modelling. Cambridge: University Press, 2010 (1st ed.).

GUTENBERG, BENO; RICHTER, CHARLES F. Seismicity of the Earth and associated phenomena. Princeton: University Press, 1949.

KING, GEOFFREY C. P.; STEIN, ROSS S.; LIN, JIAN. Static stress changes and the triggering of earthquakes. Bull. Seismol. Soc. Am., 2, março 1994.

LEI, LI. Understanding seismic body waves retrieved from noise correlations: toward a passive deep Earth imaging. Un. Grenoble Alpes, 2018 (tese).

METOIS, MARIANNE. Quantification du couplage au long de la subduction chilienne. Paris: Inst. Phys. du Globe (IPGP), 2012.

OGATA, Y.; KATSURA, K. Analysis of temporal and spatial heterogeneity of magnitude frequency distribution inferred from earthquake catalogues. Geophys. Journal Int. 113: 727-738, 1993.

ROTHÉ, J. P. The seismicity of the Earth / La seismicité du globe 1953-1965. Paris: UNESCO, 1969.

RYAN, JAMIE; BECK, SUSAN; ZANDT, GEORGE; WAGNER, LARA; MINAYA, ESTELA; TAVERA, HERNADO. Central Andean crustal structure from receiver function analysis. Tectonophysics 682(6): 120-133, 2016.

SITHARAM, T. G.; JAMES, N.; KOLATHAYAR, S. Earthquake and seismicity: comprehensive seismic zonation schemes for regions at different scales. Springer: Cham, 2018.

SOBOLEV, S. V.; BABEYKO, A. Y. What drives orogeny in the Andes? Geology 33(8): 617-620, 2005 . 
STEIN, SETH \& WYSESSION, MICHAEL. An introduction to seismology, earthquakes, and Earth structure. London: Blackwell, 498pp.

STERN, CHARLES R. Active Andean volcanism: its geologic and tectonic setting. Revista Geológica de Chile 31(2): 161-206, 2004.

TURIENZO, MARTÍN; DIMIERI, LUIS; FRISICALE, CRISTINA; ARAUJO, VANESA; SÁNCHEZ, NATALIA. Cenozoic structural evolution of the Argentinean Andes at 34040'S: a close relationship between thick and thin-skinned deformation. Andean Geology 39(2): 317-357, 2012.

ZALIAPIN, ILYA; BEN-ZION, YEHUDA. Earthquake declustering using the nearest-neighbor approach in space-time-magnitude domain. Journal of Geophysical Research: Solid Earth 125(1), 2020.

UDÍAS, AGUSTÍN; BUFORN, ELISA. Regional stresses along the Eurasia-Africa plate boundary derived from focal mechanisms of large earthquakes. In: UDÍAS, AGUSTÍN; BUFORN, ELISA. Source mechanism and seismotectonics. Basel: Springer, 1991. p. 433-448.

UTSU, T.; OGATA, Y; MATSU'URA, R. S. The centenary of the Omori formula fora a decay law of aftershock activity. J. Phys. Earth 43: 1-33, 1995.

Trabalho enviado em $08 / 10 / 2019$

Trabalho aceito em 10/12/2020 